\title{
La Saga de L'Acadie
}

\author{
Robert Viau \\ Université du Nouveau-Brunswick
}

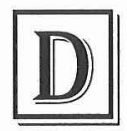

epuis une vingtaine d'années, les romans historiques sur l'Acadie et la Déportation foisonnent. Les premières ouvres qui portaient sur ce thème n'étaient que plaquettes, mais au fil des ans celles-ci sont devenues des pavés de plusieurs centaines de pages. Les romansfleuves de Claude Le Bouthillier, Jacques Gauthier et Pierre Verville ressuscitent dans des volumes massifs la culture et le passé de l'Acadie, décrivent la résistance acharnée de personnages exemplaires et leur volonté de "s'ancrer dans le roc de leur pays» (Le Bouthillier 1994, 108). Certes, le lecteur s'empêtre trop souvent dans la lourdeur des intrigues qui n'en finissent plus de s'éterniser, tout comme la vie des héros d'ailleurs, mais comment faire autrement dans une ouvre qui se propose de décrire la fondation d'un pays et le sort de familles acadiennes éparpillées aux quatre coins du globe, luttant constamment pour survivre et transmettre leur langue, leur culture et leur foi? Oscillant entre les histoires de chacun et la grande Histoire, ces romanciers nous brossent un tableau saisissant de l'épopée acadienne.

Depuis le début du nouveau millénaire, d'autres romans imposants sur la Déportation pèsent sur les présentoirs des librairies. Quelques-uns sont de facture supérieure. Ainsi, le Français Alain Dubos a récemment publié une véritable saga de quelque deux mille trois cents pages sur l'aventure américaine de la famille Lestang et leurs descendants acadiens. Lire cette ouvre, c'est découvrir l'histoire de l'Acadie. 
Acadie, terre promise

L'Acadie, c'est la France des hommes libres.

(Dubos 2002, 282)

Dans la cale d'un navire qui " puait trop la misère, la chiasse, l'haleine des malades " (18), des civils embarqués prient désespérément afin de survivre à une bourrasque. Ils cherchent la présence divine dans un espace restreint d'où s'échappe la " senteur fétide d'humanité souffreteuse » (18). Où sommes-nous? Dans un navire qui transporte des déportés de Grand-Pré? Alain Dubos entame sa saga de la famille Lestang par une scène qui annonce ce qui surviendra quelques centaines de pages plus loin. Il ne s'agit pas de la Déportation, mais de l'arrivée d'immigrants du Béarn qui veulent refaire leur vie en Acadie, bien qu'ils ne soient pas assurés d'atteindre bon port. Alain Dubos, ancien vice-président de l'organisation Médecins sans frontières, prix Nobel de la paix, et auteur de romans qui se déroulent sur les terres landaises et dans le Sud-Ouest de la France, rend un hommage bouleversant à ces immigrants français qui ont fondé l'Acadie, subi la Déportation et lutté afin que l'Acadie connaisse une singulière résurrection.

Que représente l'Acadie pour ces Français souffrant du mal de mer sinon, comme l'indique le titre, une " terre promise ". Dans ce monde inconnu, immense, de forêts, de lacs, de rivières et de tribus amérindiennes, ils découvrent une riche terre qui livre ses fruits avec générosité : "Tout était différent, ici, le paysage, les hommes, le ciel, même, ouvert aux quatre vents comme autant de portes sur l'avenir» (68). Le roi Louis XIV est loin et les seigneurs du pays peu exigeants, mais en revanche la petite colonie française perdue dans le coin nord-est de l'Amérique, accrochée à quelques fortins plus ou moins solides, est constamment " menacée par des prédateurs anglais sans scrupules " (25). Comme le rappelle Jacques Hébert, un des premiers colons : " méfie-toi toujours des Anglais et de leurs colons, par n'importe quel temps " (32). Le décor est certes grandiose, mais les villages acadiens disséminés le long de la baie Française (baie de Fundy) donnent un sentiment de précarité qui laisse prévoir le possible destin de la colonie. Dans cette Acadie dont la devise pourrait être "Étrange pays, paix impossible » (38), le patriarche Jean Lestang espère pouvoir nourrir sa famille à condition que la guerre ne vienne pas de nouveau tout bouleverser.

En quelques pages, Dubos esquisse l'attrait que présentait l'Acadie pour les Français du XVII ${ }^{\mathrm{e}}$ siècle, mais aussi ce qu'elle évoque pour les 
Français du XXI ${ }^{e}$ siècle. Cette terre de cocagne attire nos cousins d'outremer en quête de vastes espaces vierges et ceux-ci ne peuvent oublier qu'elle leur serait fidèle, si Versailles ne l'avait pas dédaignée. Il s'agit ici de cette Acadie des débuts, de "l'empremier ", une province lointaine de France peuplée par des Poitevins, des Angevins et des Béarnais qui n'hésitent pas à boire "Au roi de France » ou « Â notre pays de France » (71). Le regard qu'ils posent sur ce nouveau continent est celui d'étrangers pas encore acclimatés, pas tout à fait Acadiens. Leurs références sont européennes, leur vision du monde celle d'anciens métayers, descendants de serfs. Il y a ici une vue extérieure sur ce qui s'est déroulé en Acadie et sur ce qui aurait pu se dérouler. Cela nous vaut des pages sur le fonctionnement des aboiteaux, sur la beauté du site entourant Port-Royal, sur la singularité des coutumes amérindiennes, etc.

Posséder la terre a de quoi séduire, mais les Lestang se retrouvent non pas à Port-Royal mais à Pentagouët (dans le Maine actuel), au fond d'un fort peuplé de soldats dépenaillés et de "Sauvages » inquiétants. De plus, ces arrivants doivent affronter deux nouveaux ennemis : les rigueurs d'un climat sans pitié, surtout en hiver, et la présence menaçante d'un ennemi supérieur en armes et en nombre. L'hiver pour les paysans est tout « autour d'eux, blanc et glacé, comme un suaire sous lequel ils étouffaient " (236), tandis que les Anglais demeurent une présence hostile qui à tout instant peut fondre sur la petite colonie et la dévaster.

Dans de telles circonstances, pourquoi rester? que cherchent ces colons français en Acadie? un bien inestimable : la liberté, l'affranchissement des servitudes, dans « un pays nouveau où la liberté des hommes semblait aux dimensions de la forêt et de l'océan " (127). Les enfants de Jean sont conscients qu'en Amérique les rapports sociaux ne seront plus tout à fait les mêmes et ils se font le serment de ne jamais subir le sort des manants et des métayers du Béarn et du royaume de France. Nulle question de courber le dos devant les maitres, car " l'Acadie, c'est la France des hommes libres " (282). Et si vivre à l'ombre des places fortes d'Acadie ne vaut pas mieux que besogner la terre au pied des forteresses françaises, ils jurent de s'en aller ailleurs, plus loin pour trouver la liberté qui leur a été promise. Ces Français d'Amérique ne seront jamais comme leurs ancêtres, soumis aux caprices des seigneurs, mais connaitront une liberté à la mesure de ce nouveau continent, à la mesure du nouveau peuple auquel ils s'intègrent.

Afin de mener à bien ce récit sur la grande aventure américaine, les enfants de Jean Lestang incarnent chacun à leur tour un des mythes fondateurs acadiens. Julien se fond dans le monde sauvage des chasseurs et des Amérindiens, comme l'a fait le baron de Saint-Castin. Pierre, tel le 
corsaire Morpain, choisit la mer, tandis que Catherine vit des amours passionnées et assure la continuité de la lignée. Le coureur des bois, le corsaire et la femme forte font un roman plus mouvementé que le récit du laboureur qui sème et plante un pays.

Julien rappelle cette classe d'hommes qui attirés par la nature sauvage deviennent davantage des Amérindiens que des Français, et se fondent dans la société des " hommes libres" (134). Ayant perdu une main lors du siège du fort Pemaquid, Julien rêve à des voyages sans fin, le long des rivières, loin des forts et de la guerre. L'offrande d'une main à ces affaires qui ne le concernaient pas suffisent pour l'affranchir de toute loyauté (d'ailleurs que signifie ce mot dans les forêts où il n'y a pas de règles de jeu?) et il n'hésite pas à échanger des fourrures avec des commerçants anglais ni à vivre parmi eux.

Alors que Julien rêve à l'immensité de la forêt, Pierre est attiré par l'immensité de l'océan. Il n'est pas intéressé au quotidien des colons acadiens qui vivent le dos tourné à la mer et bâtissent des digues contre elle. Pierre s'embarque avec le corsaire Bastien Le Dantec, surnommé le Bellilois, et devient à son tour pêcheur en temps de paix, corsaire en temps de guerre. Catherine, à la suite d'amours impossibles, connaît « la hantise de devoir continuer à vivre dans cet espace borné par une mer grise et de sombres forêts " (208). Telle Maria Chapdelaine, elle épousera un cultivateur, ayant perdu l'aventurier qu'elle aimait. Avec cet homme, Jacques Hébert, elle aura trois enfants qui continueront la lignée des Lestang.

Dans les romans de Dubos défilent sans interruption des militaires, des coureurs des bois, des Amérindiens, des missionnaires et d'autres personnages mythiques. Pourtant, ce n'est pas ainsi qu'une colonie est peuplée et l'auteur rappelle l'existence de personnages moins exotiques, mais essentiels, les colons. Certes, les Saint-Castin et les d'Iberville font connaitre la gloire du roi, mais ces victoires sont sans lendemain : " ces hardis coups de main avaient de la gueule mais c'était tout, hélas » (251). Les laboureurs de Port-Royal jouent un rôle autrement plus important, fondent des familles, partagent leur bonheur simple avec leurs voisins et augmentent leurs minuscules domaines avec l'opiniâtreté des fourmis. Ce sont ces êtres courbés sur le bois de leur soc qui sont les véritables fondateurs de l'Acadie, les " obscurs, les courbés, les gratteurs aux ongles noirs, aux cheveux raides de crasse et de poussière » (252). Ces Français, qui étaient rien ou presque, ont commencé à construire un pays au rythme des récoltes et des naissances.

Mais l'Histoire avec un $\mathrm{H}$ majuscule reprend ses droits et à la suite des revers des armées du vieux roi de France revient cette question angoissante : 
" comment demeurer Français au Canada quand l'adversaire amassait, avec infiniment de patience, d'obstination et de haine vigilante, les hommes et les armes de sa future conquête " (268). Après les guerres d'escarmouches de la Penobscot viennent les guerres menées de front avec troupes régulières, artillerie et vaisseaux de ligne. La France a abandonné sa colonie et à Port-Royal le fort de M. de Subercase, pourri, vermoulu, tombe en ruine, "à l'image de la France tout entière " (410). Le siège terminé, l'Acadie abandonnée à la suite de la signature du traité d'Utrecht en 1713, les miliciens redeviennent des laboureurs. Il faut vaquer à des occupations moins glorieuses, tout en s'adaptant au nouveau régime : "La neige recouvrait tout, champs et chaumes, collines et rivages. Il fallait montrer que l'on restait unis, pour s'encourager et continuer à vivre dans l'hiver et sous une nouvelle autorité " (434).

Et la Déportation? Ce drame historique hante le récit telle une malédiction et de nombreux épisodes préfigurent ce qui doit arriver. Dès les premières pages du roman, Jacques Hébert se souvient des premières attaques anglaises, "des églises profanées au milieu des bourgs dévastées, des étables vidées de leur bétail », et voit dans ces ruines « le possible destin de la colonie " (36). Lors d'une rencontre avec le colonel Church, le gouverneur du Massachusetts suggère de " pousser cette engeance papiste vers la mer " (126) et de la remplacer par de bons paysans anglais. À la suite de la chute de Port-Royal, la menace d'une déportation et de l'arrivée de colons protestants sur les terres acadiennes se fait de plus en plus sentir. Les Acadiens deviendront-ils des étrangers sur leur terre natale?

Dans ce cas, que faire? se résigner ou poursuivre la lutte? Face à l'incertitude des temps à venir, beaucoup penchent pour que l'on cesse de résister au nom d'un roi oublieux, d'autres s'apprêtent à combattre encore. Dubos illustre ce conflit qui divisa les Acadiens en démontrant les hésitations de la troisième génération des Lestang. Jérôme, le fils de Catherine, a hérité du drapeau français qui flottait à Port-Royal et pour lui, il n'est pas question de trahir le roi de France. En revanche, l'exaltation de la jeunesse n'est guère approuvée par les colons dont les champs, les digues, les vergers sont les seuls biens que Dieu leur ait accordés : « leur sauvegarde est plus importante que tout le reste» (470).

Dubos décrit le souci, l'angoisse, l'incertitude des temps à venir que ressentent les Acadiens. De 1710 à 1755, les mêmes projets de libérer la colonie, les mêmes espérances, les mêmes prières s'élèvent vers un Dieu qui semble indifférent aux malheurs des Acadiens. Les gouverneurs anglais tolèrent ces papistes afin qu'ils continuent à nourrir les troupes de sa majesté. Certes, on tente par tous les moyens de leur faire prêter un 
serment d'allégeance, mais les Acadiens se défilent. Ils profitent de la paix pour étendre leurs champs et accroittre leur famille : "La paix, cela voulait dire aussi de l'espace et de quoi manger pour tous, avec, au fond des âmes et des cœurs, enfouie comme une braise sous la cendre, l'espérance qu'un jour le rouge des vestes anglaises laisserait place au bleu de France " (480).

Les petits-enfants de Jean Lestang ont eux aussi " le vertige de l'Amérique " (474) et ils s'éloignent de Port-Royal pour cultiver les nouvelles terres de Pisiquid ou participer à la fondation de Louisbourg. Certains sont liés à leur petit carré de blé, de lin et de pommiers, d'autres se drapent dans la bannière de la France, quelques-uns prisent un peu trop les pièces de monnaie à l'effigie du roi George. Tous tentent de survivre à une époque où la méfiance et l'incertitude peuvent mener à de nouveaux drames. Cependant, Dubos est français et il ne cesse de rappeler à ses frères d'Amérique qu'ils doivent demeurer dans la fidélité du vieux pays de France : «Ne jamais oublier qui l'on est et d'où l'on vient » (494). L'auteur va même jusqu'à évoquer Mascarène et Doucett, huguenots ralliés à l'Angleterre, " deux traîtres, aux yeux de Jérôme Hébert, qui y décelait comme certains autres un piège mortel pour les Français d'Amérique " (498). Bon sang ne peut mentir et les Acadiens ne doivent pas suivre l'exemple de ces apostats. Enfin, comment interpréter ces paroles de résistance : «Les guerres se continuent par d'autres voies », disait Pierre. Et il ajoutait, sibyllin : "Les guerres ne se terminent jamais » (645)? Dubos, et il n'est pas le seul auteur français à écrire ainsi, refuse de croire à la disparition des liens entre l'Acadie et la France, et à une époque future où la France «ne serait plus qu'une lointaine marraine et ses provinces des gravures jaunies, au fond d'une mémoire en fuite » (719). Ce ton, ce regret nostalgique que la France n'ait pas mieux défendu ses colonies, nous le retrouvons dans les romans de Léon Ville et les ouvrages historiques d'Émile Lauvrière, tous deux Français.

Mais la question se pose : jusqu’à quel point ces colons nés en Acadie ont-ils conservé cette fidélité envers une métropole lointaine qui ne leur a apporté que guerres et misères? Les Acadiens connaissaient la prospérité et l'indépendance en terre d'Acadie. Passant constamment d'une monarchie à une autre, ne participant pas aux conflits européens, ils avaient acquis une certaine liberté d'action et, avec une sagesse toute voltairienne, plantaient leurs choux en toute quiétude. Comme le rappelle Antonine Maillet :

Car l'Acadie, à force d'être ballottée d'un maittre à l'autre, avait fini par se faufiler entre les deux, par les leurrer tous et par mener ses affaires toute seule, juste sous le nez des Louis et des George des vieux pays qui 
reniflaient encore du côté des épices. Et sans souffler mot, la petite colonie d'Atlantique laissait les rois de France et d'Angleterre se renvoyer des cartes revues et corrigées d'Acadie et de la Nova Scotia, pendant qu'elle continuait allègrement à planter ses choux. (Maillet 1990, 19)

Dans les récits traditionnels de la Déportation rédigés par les Acadiens et les Québécois, les Acadiens n'ont qu'une patrie : l'Acadie, mais pour un romancier français, tel Dubos, il est préférable de souligner la fidélité des cousins d'Amérique à la métropole.

Alain Dubos évoque les grands moments de l'histoire de l'Acadie, mais le roman historique n'est pas la simple transposition de documents. Dans Acadie, terre promise, le destin de personnages d'origines diverses se croisent et il en résulte des aventures surprenantes. Nobles et roturières, Amérindiennes et corsaires se rencontrent, s'aiment et se séparent. Une trahison est vengée dans le sang trente ans plus tard. L'enfant illégitime de Pentagouët côtoie sans le savoir son demi-frère, aristocrate de Beaubassin, et enfante à son tour un bâtard à demi fou, enfermé dans une riche maison de Boston. Les frontières n'ont plus de sens alors que la lignée des Lestang s'étend à toute l'Amérique.

La mentalité des descendants de Français évolue elle aussi. En ces temps de paix difficile, les Acadiens se méfient des promesses des militaires, tant anglais que français. Lors de leurs veillées, ils se remémorent la litanie des erreurs, des occasions manquées, des trahisons et des quelques miracles insuffisants pour sauver l'Acadie française. Face à un avenir incertain, ils préferent ne compter que sur eux-mêmes et rester neutres; refuser le serment d'allégeance tout en côtoyant l'Anglais et en composant avec lui. Leur opinion se résume donc à un profond désir de labourer, de semer et de moissonner en paix les terres durement acquises sur la mer, et de connaitre la joie tranquille de voir croître leurs familles et leurs troupeaux.

Jérôme lui parla de ses sœurs, réunies par la guerre à Grand-Pré, de leurs maris qui moissonneraient bientôt de l'or sur les terres les plus riches d'Amérique. C'était la douce Acadie, le paradis donné aux hommes et aux femmes qui le méritaient, parce qu'ils avaient su le mettre en valeur. Pierre hochait la tête. La douce Acadie, certes! Et le paradis pour un peuple simplement heureux d'y vivre. Mais pour combien de temps? (650)

Malgré la paix précaire, "il faut vivre, tant que l'on en a encore le temps " (534). D'un côté, il y a ces êtres sûrs de leurs droits, qu'un siècle d'existence 
en Acadie a mis en confiance, de l'autre, la rage anglaise de tout contrôler, de la Géorgie à la baie d'Hudson.

Dans la dernière partie d'Acadie, terre promise, Dubos décrit les déportations des Acadiens en 1755, triste épisode pendant lequel tout ne fut " que calcul, détermination froide, absence totale de pitié " (845). À titre d'ancien vice-président de l'organisation Médecins sans frontières, il a exercé la médecine humanitaire dans tous les points chauds du globe et il s'attache à décrire dans son roman la souffrance des innocents qui subissent la guerre. Il rend un hommage bouleversant aux victimes de la Déportation et il stigmatise ceux qui exécutent des crimes contre l'humanité en se cachant sous le paravent des ordres reçus. Lors de l'embarquement des femmes et des enfants acadiens, il met en relief : " le crime absolu, longtemps caché derrière les ordres des officiers, la discipline nécessaire, le discours haineux ou indifférent de bien des soldats ». Comme le résume un personnage : "On assassinait sous ses yeux un peuple» (947).

Dans ce roman d'une très grande rigueur historique, nous notons tout de même des erreurs. L'auteur écrit que le curé de Saint-Charles a été expulsé de son presbytère fin août, que les Acadiens de Grand-Pré possédaient encore des fusils et qu'ils auraient pu résister " armes à la main " (821), que les Micmacs ont averti les Acadiens de ce qui s'était passé à Halifax et à Beauséjour, que Lawrence a annoncé aux délégués acadiens que la France avait déclaré la guerre, que les Acadiens de Beauséjour ont été embarqués aussitôt qu'ils ont été faits prisonniers, que ceux de Pisiquid ont été conduits sous escorte militaire pour entendre la lecture de l'ordre de déportation, que Monckton a sévi à Annapolis Royal, que Winslow était un major, etc., mais n'ergotons pas sur des vétilles. Dubos explique bien cette " dialectique de prudence " (823) par laquelle les Acadiens des Mines ont accepté de se rendre à l'église afin d'entendre la proclamation royale. Succèdent à des hésitations et des pourparlers inutiles les moments tragiques de la Déportation : le 5 septembre 1755, la lecture de l'ordre de déportation par le lieutenant-colonel Winslow aux Acadiens réunis dans l'église de Grand-Pré; le 10 septembre, l'embarquement des jeunes Acadiens à la pointe des baïonnettes au milieu des pleurs et des prières; le 8 octobre, l'embarquement des vieillards, des malades, des femmes et des enfants dans une scène où la confusion se mêlait au désespoir et à la désolation. Des enfants et des parents, des mères et des filles, des frères et des sœurs, des fiancés et des fiancées, des amis qui, ne croyaient se quitter que pour quelques jours, se séparent pour ne plus jamais se revoir.

L'épisode de la déportation des Acadiens de Grand-Pré est en quelque sorte la pierre de touche qui permet de juger des intentions d'un auteur. 
Pour certains auteurs, dont le plus connu est certainement Henry Wadsworth Longfellow, c'est l'occasion de souligner l'innocence et la résignation chrétienne des Acadiens. Pour d'autres, dont Dubos, il s'agit de démontrer que les Acadiens n'étaient pas aussi moutons que l'histoire les a dépeints généralement, en donnant de la Déportation une interprétation moins veule, plus virile. Ainsi, Jérôme Hébert abat d'un coup de pistolet le major Potter; Jacques Hébert, un enfant faible et maladif, refuse d'obéir aux Anglais et crie vengeance; sa mère, Isabelle Le Dantec, apostrophe Winslow et lui lance une poignée de vase; Thomas Hébert rejoint les Micmacs et prépare la résistance; Anne-Marie Melanson préfere la déportation avec les siens à un mariage avec un soldat écossais. Le désir de survivre et de se venger motive les déportés. Comme le souligne Jacques Hébert :

De l'église au Hannah, il s'était fait des amis, des frères, pour remplacer ceux qu'il avait perdus. Et il vivait, avec assez de haine au cœur, à l'âme et jusqu'au bout du plus infime de ses muscles, pour être déjà certain qu'il survivrait, tant qu'il n'aurait pas assouvi son besoin de vengeance. (966)

De la splendeur guerrière des "Sauvages " aux vaillants exploits des corsaires et miliciens acadiens, Dubos dépeint dans Acadie, terre promise les riches heures de la colonie française, ses années de gloire et ses années de misère. La première partie, "Les émigrants ", éveille ce rêve de vastes espaces qui hante la littérature française et qu'a su exploiter Louis Hémon, la deuxième, "Les Acadiens ", rappelle que les Acadiens sont des Français d'Amérique qui n'ont jamais renié leurs origines, tandis que la dernière partie, "Les Maudits ", évoque pour une dernière fois la société acadienne que l'on rayait d'un coup de baïonnette. Mais si l'Acadie a disparu, les Acadiens en tant que peuple n'avaient pas fini de faire parler d'eux et Dubos raconte leur refus de disparaître dans un deuxième roman.

\section{Retour en Acadie}

[...] les Anglais avaient pourchassé un peu partout ce qui restait d'Acadiens, empli quelques cales de plus et abandonné le terrain aux corbeaux et aux busards.

(Dubos 2003, 111)

Dans l'ouvre d'Alain Dubos, chaque personnage, chaque génération est confrontée à une problématique particulière : l'adaptation à la forêt, la 
mise en culture des marais, la découverte de sociétés radicalement différentes et la survie aux destructions de la guerre. Le très grand nombre de membres de la quatrième génération des Lestang-Hébert-Melanson permet à l'auteur de décrire les séquelles de la Déportation et le débarquement des Acadiens dans les ports américains. C'est dans ses pages que l'auteur révèle son expérience de guerre. Que l'on soit dans les rizières du Cambodge ou les hauts plateaux de l'Afghanistan, les civils entassés dans des camps de misère souffrent des mêmes maux : la soif, la faim, la maladie, la disparition de membres de la famille, la menace de nouvelles violences et la mort, toujours présente, qui rôde autour des réfugiés et " chois[it] ses proies avec des caprices de divinité en colère » (47). L'auteur établit des liens avec d'autres campagnes d'extermination et l'on ne peut lire sans frémir des passages comme celui-ci : « cette obstination à parfaire le mal, à remplir les vides au bas des feuilles de recensement. Des gens aussi impeccablement précis, comptabilisant les besoins des déportés à la demilivre de lard près » (366). L'homme n'apprend pas de ses erreurs et la destruction d'une société, qu'elle ait eu lieu en 1755, en 1945 ou en 2005, engendre les mêmes horreurs.

En 1755, écrit Dubos, les navires affrétés à la déportation, impropres au transport d'une cargaison humaine, ont été surchargés d'hommes, de femmes et d'enfants, souvent séparés de leurs familles. En mer, les tempêtes, le manque d'eau et de nourriture, l'entassement ainsi que les mauvaises conditions sanitaires et les maladies font que plusieurs navires perdent plus du tiers de leurs passagers. Bon nombre de vieillards et d'Acadiens affaiblis trouvent dans la mort la fin de leurs misères. Dispersés du Massachusetts à la Géorgie, les Acadiens affrontent l'accueil hostile des colons américains, en majorité protestants et anti-catholiques. Ceux-ci se plaignent de ne pas avoir été prévenus de l'arrivée des Acadiens et de la surcharge de dépenses que représente l'aide accordée par l'État à ces indigents. De plus, la guerre ayant été officiellement déclarée, la présence des Acadiens suscite la méfiance et la haine. De chapitre en chapitre, Dubos nous transporte d'une colonie à une autre, d'un groupe de déportés à un autre. Nous quittons les chaleurs moites de la Caroline pour revenir dans l'hiver mordant de l'Acadie, nous délaissons les esclaves du Sud, dont le sort peu enviable est comparé à celui des Acadiens, pour retrouver les Puritains de Boston, empesés dans leurs préjugés.

En Caroline du Sud, les Acadiens travaillent dans des rizières au côté d'esclaves noirs, mais le gouverneur leur permet de quitter l'État car il ne veut pas de cette engeance. Sur une frêle embarcation, les déportés qui ont survécu à la malaria, à la dysenterie et au désespoir voguent vers le nord, 
ayant comme capitaine, Isabelle Le Dantec, la fille d'un corsaire. Cette nouvelle Pélagie-la-Charrette recueille des retailles du peuple acadien au Maryland et poursuit sa route par mer et par terre jusqu'à l'île Saint-Jean où elle retrouve son époux et deux de ses enfants.

En Virginie, on refuse que les Acadiens débarquent dans les ports de l'État et on les refoule en Angleterre. Jean Melanson et son épouse Madeleine, devenue folle, sont emportés par la dysenterie. La sœur de Madeleine, Marguerite, et sa fille Cécile, de même que ses neveux François et Jean-Pierre traversent l'océan. Ramenés en France à la suite du traité de Paris, ils y sont restés dix ans avant de partir peupler la Louisiane.

Anne-Marie Melanson s'enfuit du Neptune grâce à William Jeffries qui, dégoûté par les événements de Grand-Pré, a quitté l'armée britannique. L'Acadienne épouse William et s'installe dans la riche demeure des Jeffries à Boston où elle fait tout en son pouvoir pour secourir ses concitoyens déportés au Massachusetts. Dans cet État, puisque les Acadiens sont à la charge du gouvernement, les enfants sont enlevés et placés chez des Américains aisés; les parents désespérés présentent des pétitions pathétiques aux autorités pour les retrouver. Le sort de nombreux Acadiens, " liés par contrat " à des maîtres anglais, assignés à un territoire qu'ils ne peuvent quitter sous peine d'emprisonnement ou de sévices physiques (mis au supplice du bloc ou fouettés publiquement), n'est guère mieux que celui des esclaves.

Jacques et Charlotte Hébert, de même que leurs cousins Antoine, Gilles et Pierre Melanson, se retrouvent à Philadelphie où une épidémie de variole emporte un tiers des déportés. Le linge des mourants est ensuite confisqué et offert aux "Sauvages » de l'Ouest afin qu'ils soient à leur tour décimés par la maladie. Plutôt que rester parqués avec les autres Acadiens dans une grange du port, Jacques et ses cousins acceptent de devenir valets de ferme pour des mennonites et " couaquères " (147). Mais les garçons ne s'adaptent pas à leur nouvelle condition. Si Charlotte, défigurée par la variole, décide de rester chez les mennonites, Jacques ne pense qu'à s'enfuir. Traversant les Appalaches, il est recueilli par des Tuscaroras qui eux aussi ont été massacrés et chassés de leurs terres. Il parvient enfin à atteindre le fort Duquesne où il s'engage à titre d'éclaireur dans l'armée française. Voulant à tout prix se venger, Jacques combattra les Anglais dans la région des Grands Lacs avant de participer à la bataille des plaines d'Abraham.

Pendant ce temps, des Acadiens qui ont réussi à échapper à la Déportation se cachent dans les forêts de ce qui était l'Acadie. Jérôme Hébert et le jeune orphelin Antoine Trahan se réfugient chez les Micmacs avant de se rendre à Louisbourg, où Jérôme retrouve son beau-père, Pierre 
Lestang. Le vieux corsaire met son navire à la disposition des survivants de la Déportation et transporte ceux qui le souhaitent à l'Île Saint-Jean. C'est ainsi qu'il retrouve ses petits-fils, Thomas et Baptiste, qui ont rejoint les camps de réfugiés du lieutenant Charles Deschamps de Boishébert sur la Miramichi. La famine, le froid, la maladie, l'épuisement physique et moral ont décimé des centaines de réfugiés du "camp de l’Espérance ». Au printemps 1757, les Acadiens qui cherchent à fuir les soldats anglais lancés à la quête de scalps n'ont plus que la peau et les os, et ont dû recourir au cannibalisme pour survivre.

Lorsque son épouse Isabelle le rejoint, Jérôme Hébert décide de quitter l'Île Saint-Jean pour Montréal afin d'éviter une seconde déportation. Le jeune Antoine Trahan, étant resté auprès de membres de sa famille, est déporté. En décembre 1758, les deux navires de transport, le Violet et le Duke William, sombrent au large de l'Espagne avec 700 Acadiens à bord. Antoine réussit miraculeusement à survivre.

Dubos n'invente aucune des horreurs qu'il décrit. Nous croyons même que son récit reste bien en deçà de la vérité. Comment décrire adéquatement le sort des Acadiens terrés dans la forêt, le sort de ceux qui cherchent à fuir en territoire français ou qui croupissent dans les geôles anglaises? Pendant sept ans, les Acadiens ont été traqués dans les bois, déportés ou tirés à vue comme du gibier. L'auteur réussit à tenir le lecteur en haleine en racontant le sort des membres de la famille HébertMelanson. Tous ne survivent pas à la Déportation : "Il manquait du monde autour d'eux, parents, frères et sœurs voguant Dieu savait où, au fond de quel mouroir. Combien faudrait-il de jours, et de nuits, pour que vînt la réparation d'un tel crime? " (42). Certains Acadiens prient avec ferveur, d'autres ne croient plus en Lui : " Disparu, le Maître Suprême, écœure, nauséeux lui aussi, du haut de son Ciel » (54). Bon nombre d'Acadiens refusent d'être absorbés dans les villes anglophones et cherchent à revenir en Acadie, d'autres sous le coup de l'humiliation et de la peur en viennent à se résigner et à accepter leur sort de proscrits. Tout dépend de l'État où le personnage a été débarqué, de son âge, de sa santé et de sa volonté de survivre.

Mais à vouloir tout raconter, l'auteur perd le lecteur, d'où certaines répliques dont l'unique but est de nous rappeler de qui l'on parle : « Je suis Thomas-à-Jérôme-à-Jacques Hébert, de la Pisiquid, cousin de Sylvain Melanson, votre paroissien de Beaubassin. Ma mère est la fille du corsaire bellilois, de la Grande-Anse » (121). L'auteur établit l'arbre généalogique des Hébert-Melanson et offre un résumé de la situation des personnages au tout début du roman. Nous devons y revenir à quelques reprises et tel les 
personnages acadiens d'Antonine Maillet devenir à l'occasion " défricheteux » de générations. Comme le précise un des personnages : " D'aucuns auraient du mal à démêler un jour les fils de cette histoire somme toute compliquée » (697), mais le récit en vaut la peine, car il offre un survol exceptionnel de l'histoire acadienne.

Certains personnages ne peuvent survivre aux années de fer et de sang et disparaissent; pour les autres, comme le souligne l'auteur, « les épreuves subies avaient creusé une faille, durci les âmes, changé les regards ", d'autres enfin pouvaient " résister à la tempête, nager dans les courants contraires de la vie, émerger des chaos" (367). Thomas Hébert qui a connu l'enfer de la Miramichi désire oublier le passé, rassembler sa famille et reconstruire son pays. Jacques Hébert qui a combattu et scalpé les Anglais refuse d'oublier et veut poursuivre la lutte armée. En 1762, les Anglais avaient embarqué plus de douze mille Acadiens, les six mille autres, disséminés entre bois, campements indiens et prisons anglaises formaient le peuple de l'Acadie. Il y avait là un mélange de familles décimées, saupoudré de quelques Acadiens passés par les États américains, le tout constituant le levain d'un nouveau peuple. Ces Acadiens remettent leur sort entre les mains de Dieu, mais ne peuvent oublier ce qu'ils ont subi : "Tous, nous gardons au fond de nous-mêmes le souvenir encore brûlant de notre solitude et de nos souffrances. Nous mourrons un jour avec cette compagnie, car nul être humain ne peut libérer son âme de pareilles cruautés " (408).

Mais ce résumé donne une fausse impression du roman. Le récit insoutenable des horreurs de la Déportation est contrebalancé par la très grande résilience du peuple acadien et son immense espoir en l'avenir. Pour les déportés, il ne s'agit pas de s'apitoyer sur leur sort, mais de réagir avec vigueur et de préparer le retour au pays natal : « Espérez, priez et d'abord battez-vous à l'intérieur de votre propre corps. [...] Luttez, mon petit, des milliers d'Acadiens font comme vous en ce moment, Dieu les garde, ils sortiront de là grandis » (154). Tout le roman exprime cette volonté de lutter et de revivre en Acadie.

On leur disait que ce pays-là n'existait plus, que jamais ils n'auraient loisir d'en récupérer la moindre parcelle. Qu'importait. On leur avait arraché le cour, les entrailles. Leurs enfants étaient morts par dizaines au fond des cales anglaises. D'autres eussent posé leur sac pour toujours et vécu là, reliques transparentes d'une société arasée. Ceux-là s'étaient mis en marche. Quelque chose de plus fort que le deuil, le chagrin, la misère, les avait portés. (227) 
Retour en Acadie est un cri du cœur, un appel d'amour à un peuple qui n'a pas fini de croître et d'étonner le monde.

Acadie, terre promise et Retour en Acadie rappellent de nombreux autres romans de la Déportation. Alain Dubos a-t-il lu les quelque soixante récits littéraires que nous avons recensés sur la Déportation? Jean et Jeanne Lestang, les fondateurs de cette dynastie de personnages, portent les mêmes prénoms que les héros de Elle et Lui, le premier roman sur la Déportation, écrit par un Acadien, le sénateur Antoine-J. Léger, et publié en Acadie, à Moncton, en 1940. Dans Elle et Lui, "Lui ", c'est Jean, un ouvrier modèle qui travaille avec courage et intelligence, prie souvent et se confie à Dieu, "Elle », c'est sa voisine Jeanne, avec qui il aura dix enfants. « Elle » et "Lui » symbolisent tous les Acadiens de cette époque.

Le jeune orphelin Antoine Trahan qui a vu disparaître sa famille à la suite de la Déportation et qui s'en remet à un étranger pour assurer sa survie évoque les aventures de Josette, dans Josette, la petite Acadienne, une orpheline qui échappe à un massacre, est recueillie par un Acadien en fuite et est conduite à un poste français. Jacques Hébert, le révolté, porte le même nom que le héros de Jacques et Marie, un des meilleurs romans historiques québécois du XIX siècle. Les deux Jacques combattent les Anglais à Québec et acceptent difficilement la victoire de leurs bourreaux. De même, l'intrigue amoureuse d'Anne-Marie Melanson et du soldat William Jeffries n'est pas sans rappeler celle du lieutenant George Gordon et de Marie Landry dans Jacques et Marie, du capitaine Scott et de Jeanne Richard dans Les Splendides Têtus ou de l'officier écossais, Archibald Cameron of Locheill et de Blanche d'Haberville dans Les Anciens Canadiens. Mais à la différence de ces personnages, William réussit à vaincre les réticences d'Anne-Marie, à l'épouser et à venger les Acadiens lors de la guerre d'Indépendance américaine.

Dans son roman, le sénateur Antoine-J. Léger souligne qu'il raconte l'histoire de ses ancêtres afin de faire progresser la cause acadienne car, comme il l'écrit : "C'est par l'histoire que l'on rapproche les générations; et mon grand-père disait que raconter le passé de la vie, c'est le faire revivre " (88). Tout comme Léger, Claude Le Bouthillier et Jacques Gauthier ont pris conscience de leur acadienneté par l'histoire et ont voulu parler de leurs ancêtres : Joseph Le Bouthillier, Joseph Gauthier... À l'instar d'Antonine Maillet, ils peuvent dire : "Pour moi, m’initier à l'écriture, c'est d'abord apprendre à déterrer mes racines " (Maillet 1976, 80). Mais Alain Dubos n'est pas acadien. Alors, pourquoi raconter ce récit? Comme l'explique un personnage : « Les Anglais ont brûlé nos églises et avec elles toutes sortes de documents. Tout est désormais dans le souvenir 
des vivants. On tue les gens, pas leur mémoire » (490). Il s'agit ici du devoir de mémoire. Dubos cherche à faire revivre le passé d'un peuple qu'il ne craint pas d'assimiler à celui de la France. Il adapte le récit de la Déportation afin de le faire connaître à ceux qui n'en ont pas encore entendu parler. Tout comme l'historien français Émile Lauvrière, il pourrait écrire : "La France a trop longtemps méconnu un peuple qui a tant souffert pour elle. Si nous pouvons par ces pages attirer sur lui l'attention qu'il mérite, obtenir une aide morale et matérielle dont il a besoin et à laquelle il a droit, nous ne regretterons rien de tout ce que nous lui avons donné de nous-même " (t.1, p. XII). En d'autres mots, il s'agit d'une part pour la France d'effacer une dette d'honneur et de renouer avec les Acadiens, les "frères retrouvés ", et, d'autre part, d'établir des comparaisons et de rappeler au lecteur que les déportations et les nettoyages ethniques de nos jours encore défraient les manchettes.

\section{La Plantation de Bois-Joli}

Si l'on plaçait un jour un emblème sur une bannière acadienne, ce serait peut-être deux mains tendues l'une vers l'autre, pour un adieu.

(Dubos 2005, 42)

Le dernier roman de la trilogie ne reprend pas les mêmes personnages, ne raconte pas ce qu'il leur est advenu, mais décrit les séquelles de la Déportation en France et en Louisiane. Après la guerre de Sept Ans, les déportés en Angleterre ${ }^{1}$, du moins les miraculés qui avaient survécu à la picote, à la dysenterie, à la longue nuit des cales et des prisons anglaises, avaient été rapatriés en France et regroupés en Bretagne, à Belle-Isle-enMer et au Poitou. Mais ces Acadiens redevenus des Français ne s'acclimatent pas à leur terre d'accueil et plusieurs décident de tenter à leur tour, un siècle et demi après leurs ancêtres pionniers, l'aventure américaine. Ils préferent s'en aller vers l'inconnu plutôt qu'être asservis comme les paysans bretons " à tant d'impôts, [et d'être] libres de rien qui valût la peine d'être vécu » (19) Au printemps de 1785, ils sont quinze cents, toutes générations mêlées, à s'embarquer à Nantes pour La Nouvelle-Orléans.

Dubos décrit ces gens qui avaient perdu tant des leurs, dans des circonstances tragiques, mais qui conservaient quelque chose d'intangible,

1 C'est-à-dire les Acadiens de la région des Mines qui avaient été déportés en Virginie en 1755, puis refoulés en Angleterre et emprisonnés jusqu’à la fin de la guerre. 
un rêve auquel ils avaient goûté, celui de posséder une terre et d'être libres. Il s'attarde sur le sort de Lalie Cormier (une descendante de Jean et Jeanne Lestang) qui s'embarque sans regrets pour la Louisiane, en quête d'" une bonne vie et de la liberté " (36). Elle quitte un passé, une blessure aussi qu'elle garde secrète. Jeune adolescente, elle a été violée par le fils d'un marchand d'esclaves, dans un vaisseau négrier de la famille. Le viol a faussé son regard sur les hommes, emmuré ses sentiments amoureux. Elle part en espérant renaître en Amérique.

La Louisiane, c'est la Nouvelle-Acadie des tropiques, des oiseaux « dont une infinité de couleurs bariolait certains envols » (54), un pays qui ressemblait à une "éponge où l'on s'enfonçait jusqu'à la taille ", des monstres « aux mâchoires plus longues que les mousquets y guettaient leurs proies humaines sous la surveillance d'aigles géants » (55). De nouveau, le lecteur subit l'attrait de régions exotiques, mystérieuses, éloignées à la fois dans l'espace et dans le temps. Mais la Louisiane, c'est aussi une société divisée entre nantis et pauvres, Créoles et Cadiens, Blancs et Noirs. Il est miné par l'esclavagisme que les Acadiens découvrent avec horreur : " J'espère que nous n'aurons jamais à fouetter l'un quelconque de ces pauvres diables. Plutôt retourner à Nantes qu'être obligé à se commettre ainsi, dit vivement Lalie » (59-60).

Pour des Acadiens qui se souviennent de la société égalitaire des Mines se retrouver en Louisiane présente une différence notable tant géographique que sociale. Lalie Cormier et les immigrants acadiens sont mal perçus par les Créoles qui se sont enrichis grâce au travail de leurs esclaves et qui se considèrent comme des aristocrates français en Amérique. Ce monde de propriétaires, tout de vanité, de suffisance et de contentement de soi, n'est pas celui des Acadiens. Pourtant, pour les nouveaux arrivants, cette Acadie du Sud est un paradis tropical, " un don de Dieu à qui savait le mériter » (128). La Louisiane abonde en poisson et en gibier, l'hiver est doux et la chasse est libre, cependant il faut se méfier des apparences. La colonie est en crise, l'indigo se vend mal, le sucre que l'on n'arrive pas à cristalliser pourrit dans les cales, des fièvres malignes conduisent incessamment à la tombe, les vers et chenilles s'attaquent aux récoltes de sorte que "la ruine est la sœur vigilante et sans pitié de la richesse " (85). À cela s'ajoute une situation politique instable.

Par une détestable habitude, la France considère le meilleur de ses colonies comme de la simple monnaie d'échange. Elle a perdu l'Acadie, puis la Nouvelle-France, et a cédé la Louisiane à l'Espagne. L'année 1763 marque la fin du rêve français en Amérique. La couronne espagnole, cousine de celle de France, encourage l'arrivée de colons acadiens et leur

\section{4 * LittéRéalité}


installation au nord de la colonie afin de parer à toute tentative américaine de s'emparer du delta du Mississippi. Dans cette colonie espagnole peuplée de Français et située au sud des États-Unis, les allégeances politiques sont précaires. Les Créoles espèrent le retour de la couronne française; les Espagnols écrasent toute tentative de révolte dans le sang, tandis que les Américains rêvent d'étendre leur domination tout le long du Mississippi. Les Acadiens, eux, ne pensent qu'à survivre.

À la différence des romans précédents, La Plantation de Bois-Joli est moins historique et plus romanesque. Certes, l'histoire mouvementée de la Louisiane est évoquée, mais celle-ci importe moins que les amours des personnages. Dans ce roman, Denis de Canisy est l'époux malheureux de Sophie de Grehan, une Créole méprisante envers tous ceux qui ne sont pas de sa caste. Denis regrette d'avoir épousé cette femme revêche et plus âgée que lui, et il s'éprend de sa cousine Lalie Cormier qui vient de s'installer sur les rives du bayou Lafourche. Celle-ci de son côté cherche à fuir Amand Boudreau qui, furieux de se voir repoussé par Lalie, a tenté de la noyer. À La Nouvelle-Orléans, Lalie connaît l'amour dans les bras de Denis et accouche d'une fille Marguerite. Il va de soi que les jeunes gens sont faits pour former un couple, d'autant plus que Sophie meurt au cours d'une tempête tropicale. Denis épouse Lalie malgré le scandale que cela provoque. Cependant, le créole continue de perdre au jeu des sommes importantes de sorte que sa plantation est menacée par la ruine. Il meurt peu après de la fièvre typhö̈de et Lalie hérite de ses biens... et de ses dettes. L'Acadienne fait face aux malheurs et la plantation est sauvée lorsqu'on découvre le moyen de cristalliser le sucre et de le rendre à cent pour cent transportable. Un âge d'or s'ouvre pour les propriétaires de plantations.

La Louisiane se partage en deux groupes francophones importants, ceux aux poignets épaissis par le travail d'une terre capricieuse et ceux avec des mains adoucies par l'oisiveté. Les Acadiens font partie du premier groupe. Ils connaissent une existence difficile, mais cela est préférable à vivre la destinée des plus humbles en France, dans un pays qui est indifférent à leur présence. La Louisiane incarne leur espérance nouvelle, leur fierté d'être libres. Malgré leur pauvreté, ils mangent à leur faim et tous rêvent de s'enrichir comme les Créoles. La caste de propriétaires créoles fait partie du deuxième groupe. Cette caste de nantis est habituée à commander à des esclaves et à tout recevoir sans travailler. Les Créoles ne songent qu'à s'amuser par le jeu, la danse et des amours illicites avec «ces femmes à la peau couleur suc de canne, fascinantes et jalousées, dont la docilité se proportionnait au désir de s'élever socialement " (227). D'autres groupes sociaux sont décrits dans le roman : les Espagnols qui gouvernent 
la colonie; les Houmas, une tribu amérindienne refoulée dans les bayous; les Américains qui sont décrits comme des rapaces qui ne pensent qu'à " étreindre, pénétrer, posséder " (88) et dont la méthode de s'enrichir sans scrupules annonce l'avenir de la colonie : " tant pis pour les petits, les perdants, les Nègres et les Indiens. Si tu n'es pas riche, c'est que tu ne le mérites pas" (189-190).

Les esclaves noirs sont l'élément caché sur lequel repose toute l'économie de la colonie. Les Créoles les considèrent comme du vulgaire bétail et les cèdent sans état d'âme pour régler des dettes de jeu. Mais les esclaves noirs fascinent les Acadiens qui se souviennent des déportés vendus à des planteurs de la Géorgie, morts d'épuisement dans les rizières de Savannah. Bien qu'elle n'apprécie pas cette façon de vivre, Lalie sait qu'elle ne peut la changer. Les quelques réformes qu'elle a implantées à la plantation de Bois-Joli sont vivement réprouvées : " ces choses-là ne se font pas, vous manquez à vos devoirs " (377). La "gâteuse des Nègres " (378) réussit tout de même à sauver de la pendaison un nègre marron et à améliorer le sort des esclaves sur la plantation : réduction des heures de travail, soins accordés aux malades et aux blessés, interdiction aux enfants de travailler la terre, etc. Mais Lalie est consciente que ces réformes sont dérisoires et que sa place n'est " assurément pas dans cet endroit où vivait un peuple sans réel statut " (513). Si à la fin du roman elle décide de quitter la Louisiane, c'est en grande partie parce que "vivre au domaine supposait qu'on en acceptât le décor humain » (443). Elle s'embarque pour la France où un certain général Bonaparte commence à se faire connaître.

Lalie apparaît comme une femme foncièrement moderne qui ne craint ni les jugements de l'Église ni les qu'en-dira-t-on de la société. Elle est consciente qu'avec le temps, les scandales seront oubliés : "La vie emportait tout, comme la crue du Mississippi » (279). La seule chose qui importe, c'est la patrie de l'amour : «Un corps, des mains, un regard; cela valait bien des frontières, des armées, des territoires à conquérir et des colonies à peupler " (548). L'observant soigner son mari, le docteur Lubet porte un jugement tout à l'honneur de l'Acadienne :

Étrange femme, pensa-t-il. Elle aurait pu vivre dans l'ombre d'un armateur ou d'un marchand, se fondre dans la tribu acadienne du bayou, vivre à la garnison avec un officier espagnol. Au lieu de quoi, elle avait accepté le scandale et l'opprobre, avant de les fuir puis de les affronter à nouveau, dans l'ambiance délétère de la plantation. Une belle âme, éclairée par une force intime d'une rare puissance. (504) 
Y aura-t-il une suite aux amours de Lalie Cormier? les dernières pages du roman laissent entendre que l'Acadienne n'a pas terminé ses pérégrinations.

Alain Dubos s'inscrit dans cette lignée d'écrivains français qui ont voulu à tout prix faire connaître la Déportation à leurs concitoyens. Les Français Huguette Perol, Jeanne Ducluzeau et Mathieu Quéméneur ont eux aussi raconté le récit des déportés acadiens, mais nul ne l'a fait avec autant de passion que Dubos, ni avec autant de minutie. Ses romans expriment à travers des destinées individuelles exemplaires les problèmes d'une époque donnée du passé et la destinée d'un peuple qu'on a voulu détruire. En cette année du $250^{\mathrm{e}}$ anniversaire de la Déportation, quiconque veut découvrir l'histoire de l'Acadie par l'intermédiaire de personnages attachants n'a qu'à lire la saga des Lestang.

Certes, il est vrai que Dubos ne peut prétendre à une exactitude parfaite même en matière de faits historiques, encore bien moins sur les points de la langue et des manières. Mais le peut-il ou plutôt le veut-il? Le romancier refuse de se confiner dans les limites de la période où se situe son récit, ce qui pourrait à la rigueur rendre ce récit totalement incompréhensible. Pour susciter un intérêt quelconque du lecteur, il est nécessaire que le sujet choisi soit en quelque sorte adapté ou traduit dans les manières et la langue de l'époque où vit le lecteur. C'est en grande partie par cet " anachronisme nécessaire » (65), selon l'expression de Lukács, que l'auteur donne vie au personnage, intéresse l'éventuel lecteur et raccorde l'œuvre avec le temps présent. À la fin du roman, le lecteur ne doit pas considérer la psychologie particulière et l'éthique qui en résultent comme des curiosités historiques, difficiles à saisir, mais doit être conscient qu'ils restent tributaires de la relation de l'auteur à son époque, à sa société. Cela explique l'importance que place l'auteur sur le nationalisme français, le rôle qu'il donne au féminisme et les multiples explications historiques et géographiques, fort utiles au lecteur contemporain, qui parsèment le récit. Par ces interférences historiques, l'auteur rattache le récit des Lestang au présent du lecteur.

Alain Dubos a refait du dedans ce que les historiens ont fait du dehors. Par l'intermédiaire de personnages fictifs, il donne un nom et un visage aux victimes et nous rappelle que les êtres humains qui ont subi la Déportation ne se réduisent pas à de simples statistiques. Il secoue le lecteur et par l'exemple du passé rappelle que de telles tragédies continuent à décimer des peuples. En fin de compte, le lecteur d'Acadie, terre promise, de Retour en Acadie et de La Plantation de Bois-Joli ne cherche pas tant une évasion dans 
le passé qu'une connaissance du passé et une explication de son présent. Ces romans historiques ne présentent pas un reflet passif du réel, mais une réflexion sur ce qui a été et sur ce qui est. Les romans d'Alain Dubos rappellent à tous la pathétique leçon du passé et ne peuvent que servir la cause de la paix dans un monde qui ne semble pas toujours avoir appris de ses erreurs.

\section{Ouvrages cités}

Aubert de Gaspé, Philippe, Les Anciens Canadiens, Montréal, Fides, 1988 [éd. originale : 1863], $410 \mathrm{p}$.

Bourassa, Napoléon, Jacques et Marie. Souvenir d'un peuple dispersé, Montréal, Fides, 1976 [éd. originale : 1865-1866], 371 p.

Desmarins, Paul [pseudonyme de Paul LeBlanc], Josette, la petite Acadienne, Montréal, Granger Frères, 1955, 125 p.

Dubos, Alain, Acadie, terre promise, Paris, Presses de la Cité, 2002, 987 p. - La Plantation de Bois-Joli, Paris, Presses de la Cité, 2005, 551 p. , Retour en Acadie, Paris, Presses de la Cité, 2003, 750 p.

Ducluzeau, Jeanne, Anne d'Acadie, Moncton, Éditions d'Acadie, 1984, $260 \mathrm{p}$.

, Le Chemin des Huit-Maisons, Moncton, Éditions d'Acadie, 1987, $359 \mathrm{p}$.

Gauthier, Jacques, Chroniques d'Acadie, Montréal, Éditions Pierre Tisseyre, 4 vol. : t.1, Clovis, 1992, 470 p.; t. 2, Oscar, 1993, 430 p.; t.3, Tranquille et Modeste, 1995, 431 p.; t.4, S'en vont chassant. 1996, $389 \mathrm{p}$.

Hémon, Louis, Maria Chapdelaine, Montréal, Fides, 1924, 215 p.

Laurent, Albert [pseudonyme], Les Splendides Têtus, Supplément de "L'Action paroissiale ", vol. 4, $\mathrm{n}^{\text {os }}$ 1-7, juin-décembre 1939, 112 p. Publié sous le titre L'Épopée tragique. Roman acadien, Montréal, Beauchemin, 1956, 255 p.

Lauvrière, Émile, La Tragédie d'un peuple. Histoire du peuple acadien de ses origines à nos jours, Paris, Librairie Henry Goulet, 1924, t. 1, 510 p.; t. 2, 596 p.

Le Bouthillier, Claude, Le Feu du mauvais temps, Montréal, Québec/Amérique, 1989, 451 p.

, Les Marées du Grand Dérangement, Montréal, Québec/Amérique, 1994, 367 p.

Léger, Antoine-J., Elle et Lui. Tragique Idylle du peuple acadien, [Moncton, Imprimerie de L'Évangéline ltée, 1940], 203 p. 
Longfellow, Henri Wadsworth, Évangéline, Moncton, Éditions PerceNeige/Écrits des Forges, 1994, 104 p.

Lukacs, George, Le Roman historique, Paris, Payot, 1965, 407 p.

Maillet, Antonine, "Expériences d'écriture. Mon pays, c'est un conte ", Études françaises, vol. 12, no 1-2, avril 1976, p. 76-83. Pélagie-la-Charrette, Montréal, Leméac, 1990, 334 p.

Perol, Huguette, Le Grand Exode de François d'Acadie, Paris, Éditions de l'amitié-G.T. Rageot, 1974, 156 p.

Quéméneur, Mathieu, L'Acadien, Sarreguemines, Éditions Pierron, 1990, $195 \mathrm{p}$.

Verville, René, Le Saule de Grand-Pré, Montréal, Fides, 2001, 486 p.

Viau, Robert, Les Grands Dérangements : la Déportation des Acadiens en littératures acadienne, québécoise et française, Beauport ( $\left.\mathrm{Q}_{\mathrm{c}}\right)$, Publications MNH, 1997, 381 p.

Ville, Léon, En Acadie, Le Martyre d'un peuple, Paris, Tolra, 1927, 157 p. En Acadie, Par le feu et par le fer, Paris, Tolra, 1927, 155 p. 


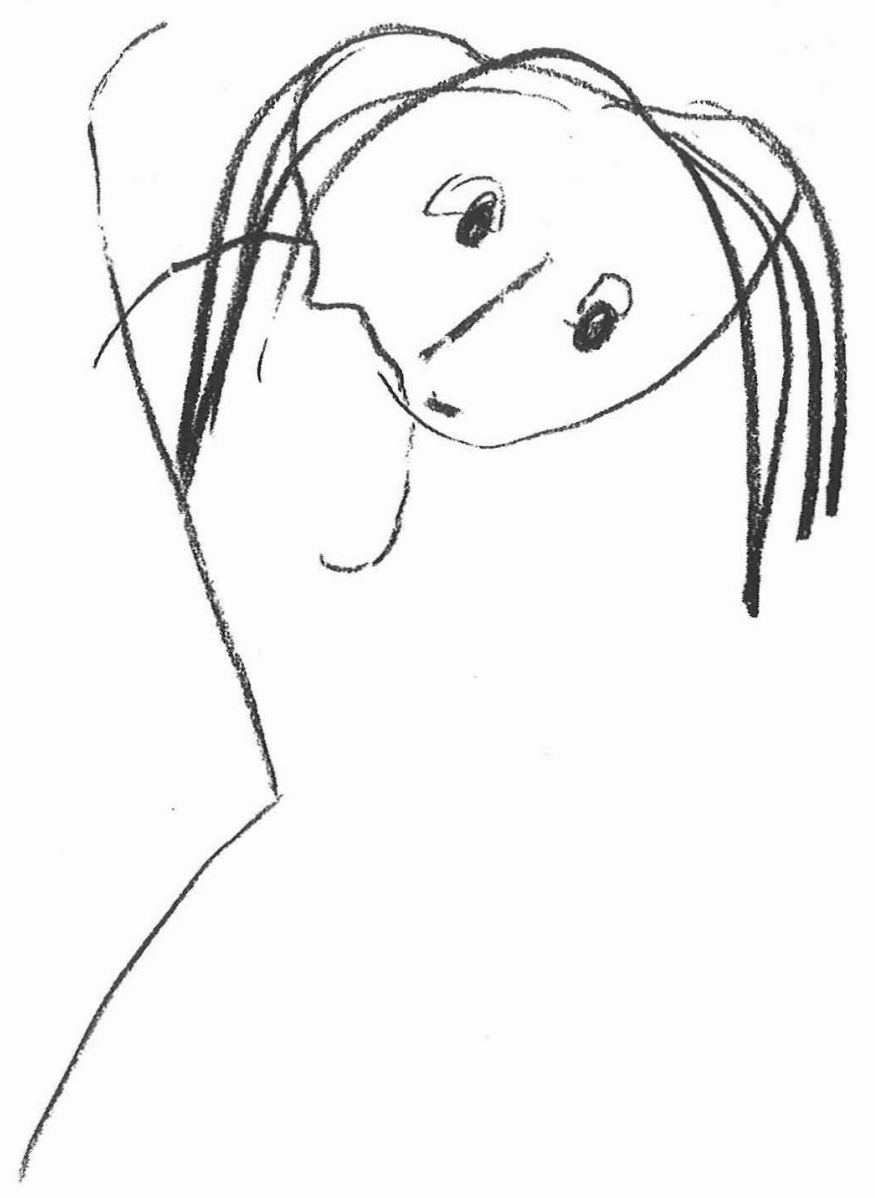

AdAm NidZgorsKI

40 - LittéRéalité 